\title{
A novel simplification of the Reynolds-Chou-Navier-Stokes turbulence equations of incompressible flow
}

\author{
Bohua Sun $^{1}$ \\ 1 Cape Peninsula University of Technology, Cape Town, South Africa*
}

\begin{abstract}
Based on author's previous work [Sun, B. The Reynolds Navier-Stokes Turbulence Equations of Incompressible Flow Are Closed Rather Than Unclosed. Preprints 2018, 2018060461 (doi: 10.20944/preprints201806.0461.v1)], this paper proposed an explicit representation of velocity fluctuation and formulated the Reynolds stress tensor in terms of the mean velocity field. The proposed closed Reynolds Navier-Stokes turbulence formulations reveal that the mean vorticity is the key source of producing turbulence.
\end{abstract}

PACS numbers: 47.27.-i,47.27.Ak,47.27.E-

Keywords: Turbulence, mean velocity, fluctuation velocity, the Reynolds stress tensor, vorticity, turbulence closure problem

\section{INTRODUCTION}

Turbulence is a difficulty subject that pervades so many aspects of peoples' daily lives [1-16]. It is believe that the turbulence flow are govern by the Navier-Stokes momentum equation is $\rho \boldsymbol{u}_{, t}+\boldsymbol{\nabla} \cdot \boldsymbol{\Pi}=0$, continuity equation of incompressible flow is $\boldsymbol{\nabla} \cdot \boldsymbol{u}=0$, where the energymoementum tensor given by $\boldsymbol{\Pi}=p \boldsymbol{I}+\rho \boldsymbol{u} \otimes \boldsymbol{u}-\mu(\boldsymbol{\nabla} \boldsymbol{u}+$ $\boldsymbol{u} \boldsymbol{\nabla})$, dynamic viscosity $\mu$, gradient operator $\boldsymbol{\nabla}=\boldsymbol{e}_{i} \partial_{i}$, base vector in the i-coordinate $\boldsymbol{e}_{i}$, and tensor product $\otimes$.

To solve the problem, in 1895 Reynolds published a seminal work on turbulence [29], in which he proposed that flow velocity $\boldsymbol{u}$ and pressure $p$ are decomposed into its time-averaged quantities, $\boldsymbol{u}^{-}, t, \bar{p}$, and fluctuating quantities, $\boldsymbol{u}^{\prime}, p^{\prime}$; thus, the Reynolds decomposition$\mathrm{s}$ are: $\boldsymbol{u}=\overline{\boldsymbol{u}}(\boldsymbol{x}, t)+\boldsymbol{u}^{\prime}(\boldsymbol{x}, t)$ and $p(\boldsymbol{x}, t)=\bar{p}(\boldsymbol{x}, t)+$ $p^{\prime}(\boldsymbol{x}, t)$, where coordinates and times are $(\boldsymbol{x}, t)$. With decomposition the Navier-Stokes equation is then transformed into Reynolds-averaged Navier - Stokes equation$\mathrm{s}$, where the Reynolds stress tensor $\boldsymbol{\tau}=-\rho \overline{\boldsymbol{u}^{\prime} \otimes \boldsymbol{u}^{\prime}}=$ $-\rho \lim _{T \rightarrow \infty} \frac{1}{T} \int_{t}^{t+T}\left(\boldsymbol{u}^{\prime} \otimes \boldsymbol{u}^{\prime}\right) d t$ is introduced, where $T$ is the period of time over which the averaging takes place and must be sufficiently large to give meaningful averages. Reynolds stress is apparent stress owing to the fluctuating velocity field $\boldsymbol{u}^{\prime}$. Since introduction of the Reynolds stress tensor, the closure problem of turbulence, namely the Reynolds equations are unclosed, has eluded scientists and mathematicians for centuries. The Reynolds equations can not be solved unless some additional restrictions are somehow determined.

Applying the Reynolds decomposition and averaging operation, we have the Reynolds equations and continuity equation of the mean velocity as follows, respectively: $\rho \overline{\boldsymbol{u}}_{, t}+\rho \boldsymbol{\nabla} \cdot(\overline{\boldsymbol{u}} \otimes \overline{\boldsymbol{u}})+\boldsymbol{\nabla} \bar{p}=\mu \boldsymbol{\nabla}^{2} \overline{\boldsymbol{u}}-\rho \boldsymbol{\nabla} \cdot\left(\overline{\boldsymbol{u}^{\prime} \otimes \boldsymbol{u}^{\prime}}\right)$ and $\boldsymbol{\nabla} \cdot \overline{\boldsymbol{u}}=0$, which is called the Reynolds (averaged) equations. For a general three-dimensional flow, there are four independent equations governing the mean velocity field; namely three components of the Reynolds equation$\mathrm{s}$ together with one mean continuity equation. However, these four equations contain more than four unknowns. In addition to $\overline{\boldsymbol{u}}$ and $\bar{p}$ (four quantities), there are also the Reynolds stresses. The Reynolds equations are unclosed. This is a manifestation of the closure problem.

In 1940 and 1945, P.-Y. Chou [30, 31] published a remarkable result and pointed out that because the NavierStokes equations are the basic dynamical equations of fluid motion, it is insufficient to consider only the mean turbulent motion. The turbulent fluctuations are as important as the mean motion and the equations for turbulent fluctuations also need to be considered. Subtracting the mean motions from the Navier-Stokes equation and continuity equation, Chou [30, 31] obtained the equations of the turbulence fluctuations $\rho \overline{\boldsymbol{u}}^{\prime}{ }_{t}+\rho \boldsymbol{\nabla} \cdot\left(\overline{\boldsymbol{u}} \otimes \boldsymbol{u}^{\prime}+\boldsymbol{u}^{\prime} \otimes \overline{\boldsymbol{u}}+\right.$ $\left.\boldsymbol{u}^{\prime} \otimes \boldsymbol{u}^{\prime}\right)+\nabla p^{\prime}=\mu \nabla^{2} \boldsymbol{u}^{\prime}+\rho \boldsymbol{\nabla} \cdot\left(\overline{\boldsymbol{u}^{\prime} \otimes \boldsymbol{u}^{\prime}}\right)$ and $\boldsymbol{\nabla} \cdot \boldsymbol{u}^{\prime}=0$. In honor of Chou's contribution, we like to propose to call the equation the Chou turbulence equation.

Although Chou [31] mentioned that the rigorous way of treating the turbulence problem is probably to solve the Reynolds' equations of mean motion and the equations of turbulent fluctuation simultaneously. However, from the presentation of [31] and all his subsequent publications [32-36], we noticed that Chou together with all other researchers $[1-8,10-16]$ did not realised that the fluctuation equations together with the mean equations already can form a closed equations system.

But researchers are making progress on understanding the physics of the Reynolds stresses. In a Preprint published on 28 June 2018 in Preprints.org, a new per- 
spectives proposed by Sun [37] could help to solve the long-standing puzzle over the turbulence closure issue. He proven that the Reynolds stress tensor is not a general second order tensor with six independent elements, while its each element is the product of two fluctuation velocity components. There are 3 velocity components in $3 \mathrm{D}$ flow, therefore the number of independent parameters is 3 rather than 6 , namely the three components of the fluctuation velocity. For three dimensional flow, we can only have three components of fluctuation velocity $\boldsymbol{u}^{\prime}$ as unknowns. It means that the Reynolds stress tensor has only three unknowns, namely $u_{1}^{\prime}, u_{2}^{\prime}, u_{3}^{\prime}$. For two dimensional flow, of course, the 2D Reynolds stress tensor has only two unknowns, namely $u_{1}^{\prime}, u_{2}^{\prime}$.

With this new understanding, Sun [37] finally shown the integral-differential equations of the Reynolds mean and fluctuation equations have same number of unknowns That is why he claim that the Reynolds Navier-Stokes turbulence equations of incompressible flow are closed rather than unclosed.

\section{THE REYNOLDS-CHOU-NAVIER-STOKES (RCNS) TURBULENCE EQUATIONS}

Sun [37] proposed the closed turbulence equations of incompressible flow. In honor of seminal contribution from both O Reynolds and P.-Y.Chou, in the future, we like to propose to call the equations as the ReynoldsChou-Navier-Stokes (RCNS) turbulence equations. The RCNS can be presented as follows

$$
\begin{aligned}
& \rho \overline{\boldsymbol{u}}_{, t}+\rho \boldsymbol{\nabla} \cdot(\overline{\boldsymbol{u}} \otimes \overline{\boldsymbol{u}})+\nabla \bar{p}=\mu \nabla^{2} \overline{\boldsymbol{u}}-\rho \lim _{T \rightarrow \infty} \frac{1}{T} \int_{t}^{t+T} \boldsymbol{\nabla} \cdot\left(\boldsymbol{u}^{\prime} \otimes \boldsymbol{u}^{\prime}\right) d t \\
& \rho \overline{\boldsymbol{u}}_{, t}^{\prime}+\rho \boldsymbol{\nabla} \cdot\left(\overline{\boldsymbol{u}} \otimes \boldsymbol{u}^{\prime}+\boldsymbol{u}^{\prime} \otimes \overline{\boldsymbol{u}}+\boldsymbol{u}^{\prime} \otimes \boldsymbol{u}^{\prime}\right)+\boldsymbol{\nabla} p^{\prime}=\mu \nabla^{2} \boldsymbol{u}^{\prime}+\rho \lim _{T \rightarrow \infty} \frac{1}{T} \int_{t}^{t+T} \boldsymbol{\nabla} \cdot\left(\boldsymbol{u}^{\prime} \otimes \boldsymbol{u}^{\prime}\right) d t, \\
& \nabla \cdot \overline{\boldsymbol{u}}=0, \\
& \nabla \cdot \boldsymbol{u}^{\prime}=0 .
\end{aligned}
$$

Denoting kinematic viscosity $\nu=\mu / \rho$, the above equations be equivalently rewritten in a conventional form

$$
\begin{aligned}
& \overline{\boldsymbol{u}}_{, t}+\overline{\boldsymbol{u}} \cdot \nabla \overline{\boldsymbol{u}}+\frac{1}{\rho} \boldsymbol{\nabla} \bar{p}=\nu \nabla^{2} \overline{\boldsymbol{u}}-\lim _{T \rightarrow \infty} \frac{1}{T} \int_{t}^{t+T} \nabla \cdot\left(\boldsymbol{u}^{\prime} \cdot \nabla \boldsymbol{u}^{\prime}\right) d t \\
& \overline{\boldsymbol{u}}_{, t}^{\prime}+\overline{\boldsymbol{u}} \cdot \boldsymbol{\nabla} \boldsymbol{u}^{\prime}+\boldsymbol{u}^{\prime} \cdot \nabla \overline{\boldsymbol{u}}+\boldsymbol{u}^{\prime} \cdot \boldsymbol{\nabla} \boldsymbol{u}^{\prime}+\frac{1}{\rho} \nabla p^{\prime}=\nu \nabla^{2} \boldsymbol{u}^{\prime}+\lim _{T \rightarrow \infty} \frac{1}{T} \int_{t}^{t+T} \nabla \cdot\left(\boldsymbol{u}^{\prime} \cdot \nabla \boldsymbol{u}^{\prime}\right) d t \\
& \nabla \cdot \overline{\boldsymbol{u}}=0 \\
& \boldsymbol{\nabla} \cdot \boldsymbol{u}^{\prime}=0
\end{aligned}
$$

Applying the divergence operation $\nabla$ on both sides of the Eqs. $(5,6)$, we can obtain equations for both mean and fluctuation pressure as follows 


$$
\begin{aligned}
& \nabla^{2} \bar{p}=-\rho \boldsymbol{\nabla} \cdot(\overline{\boldsymbol{u}} \cdot \boldsymbol{\nabla} \overline{\boldsymbol{u}})-\rho \lim _{T \rightarrow \infty} \frac{1}{T} \int_{t}^{t+T} \boldsymbol{\nabla} \cdot\left(\boldsymbol{u}^{\prime} \cdot \boldsymbol{\nabla} \boldsymbol{u}^{\prime}\right) d t \\
& \boldsymbol{\nabla}^{2} p^{\prime}=-\rho \boldsymbol{\nabla} \cdot\left[\overline{\boldsymbol{u}} \cdot \boldsymbol{\nabla} \boldsymbol{u}^{\prime}+\boldsymbol{u}^{\prime} \cdot \boldsymbol{\nabla} \overline{\boldsymbol{u}}+\boldsymbol{u}^{\prime} \cdot \boldsymbol{\nabla} \boldsymbol{u}^{\prime}\right]+\rho \lim _{T \rightarrow \infty} \frac{1}{T} \int_{t}^{t+T} \boldsymbol{\nabla} \cdot\left(\boldsymbol{u}^{\prime} \cdot \boldsymbol{\nabla} \boldsymbol{u}^{\prime}\right) d t .
\end{aligned}
$$

Although the Eqs.(1,2,3,4) are closed, if you add Eq.1 and Eq.2, the Reynolds stress tensor will be cancelled out and all equations go back to the Navier-Stokes equation; similarly the total velocity continuity equation will be restored if adding Eq.3 and Eq.4. In other words, although the turbulence equations Eqs. $(1,2,3,4)$ are closed, it would not provide us a real boost in solving turbulence problem. Nevertheless, the turbulence equations Eqs. $(1,2,3,4)$ still have an academic value, which can definitely give a better guideline in the modelling of turbulence.

\section{VELOCITY FLUCTUATION APPROXIMATION}

Although the turbulence problem can be calculated by Eqs. $(5,6,7,8)$, it would be useful to propose a fluctuation velocity without solving the equations, where the velocity fluctuation $\boldsymbol{u}^{\prime}$ satisfies the incompressibility condition $\boldsymbol{\nabla} \cdot \boldsymbol{u}^{\prime}=0$ and time average conditions $\overline{\boldsymbol{u}^{\prime}}=\lim _{T \rightarrow \infty} \frac{1}{T} \int_{t}^{t+T} \boldsymbol{u}^{\prime} d t=0$ as well.

The question is: how does one construct velocity fluctuation $\boldsymbol{u}^{\prime}$ ? Since there are no definitive stances on what turbulence is, or no scientific definition of turbulence, it is hard to guess the weight of velocity fluctuation $\boldsymbol{u}^{\prime}$ within the flow velocity $\boldsymbol{u}$. But what is apparent is that the mean field and fluctuation counterpart of velocity is interconnected, for instance, the faster $\overline{\boldsymbol{u}}$ goes, the higher the frequency $\boldsymbol{u}^{\prime}$, which implies that the velocity fluctuation $\boldsymbol{u}^{\prime}$ is dependent on the mean velocity field $\overline{\boldsymbol{u}}$. With this understanding one can propose that the velocity fluctuation $\boldsymbol{u}^{\prime}$ is a function of mean velocity $\overline{\boldsymbol{u}}$, namely $\boldsymbol{u}^{\prime}=\boldsymbol{u}^{\prime}(\overline{\boldsymbol{u}}, t)$. Therefore, the Reynolds velocity decomposition can be rewritten as $\boldsymbol{u}=\overline{\boldsymbol{u}}+\boldsymbol{u}^{\prime}(\overline{\boldsymbol{u}}, t)$. However, the closure problem would still be there if $\boldsymbol{u}^{\prime}(\overline{\boldsymbol{u}}, t)$ cannot be proposed.

Numerous observations [1-16] have shown that turbulence is caused by excessive kinetic energy in parts of a fluid flow, which overcomes the damping effect of the fluid's viscosity. Hence, turbulence is easier to create in low viscosity fluids, but more difficult in highly viscous fluids. The dynamic balance between kinetic energy and viscous damping in a fluid flow can be perceived as flow symmetries that are broken by mechanisms, which produce turbulence, and are restored by the chaotic character of the cascade to small scales [11]. This dynamic balance process is the key source to generating velocity fluctuation $\boldsymbol{u}^{\prime}$. In particular, it is found that the mean velocity vorticity $\overline{\boldsymbol{\omega}}=\boldsymbol{\nabla} \times \overline{\boldsymbol{u}}$ plays an essential role in producing turbulence. This means that velocity fluctuation $\boldsymbol{u}^{\prime}$ should be a function of both mean velocity $\overline{\boldsymbol{u}}$ and its vorticity $\overline{\boldsymbol{\omega}}$.

To satisfy the incompressibility condition $\boldsymbol{\nabla} \cdot \boldsymbol{u}^{\prime}=0$, the velocity fluctuation $\boldsymbol{u}^{\prime}$ must be divergence-free, hence we can introduce a vector function $\psi$ and let

$$
\boldsymbol{u}^{\prime}=\nabla \times \psi
$$

Based on the above understanding and mathematical requirements, we like propose the following conjecture for the vector $\psi$ :

$$
\boldsymbol{\psi}=f(\overline{\boldsymbol{u}}) \beta(\boldsymbol{x}, t)
$$

where $\beta(\boldsymbol{x}, t)$ is a scalar function and represents the nature of fluctuation. How should one determine the function $f(\overline{\boldsymbol{u}})$ ? Considering the vector as a first order tensor, if $f(\overline{\boldsymbol{u}})$ is a homogenous function of $\overline{\boldsymbol{u}}$, we should express that the function $f(\overline{\boldsymbol{u}})=\boldsymbol{b}+\alpha \overline{\boldsymbol{u}}$, in which the constant vector $\boldsymbol{a}$ can be omitted, since $\boldsymbol{\nabla} \times \boldsymbol{b}=0$. Physically, there is no velocity fluctuation $\boldsymbol{u}^{\prime}$ if there is no mean velocity $\overline{\boldsymbol{u}}$, namely $\boldsymbol{u}_{\overline{\boldsymbol{u}}=\mathbf{0}}^{\prime}=0$.

If one substitutes $\phi=0$ and Eq. (12) into Eq. (13), it will lead to the following velocity fluctuation:

$$
\boldsymbol{u}^{\prime}=\alpha \boldsymbol{\nabla} \times[\overline{\boldsymbol{u}} \beta(\boldsymbol{x}, t)]=\alpha(\beta \boldsymbol{\nabla} \times \overline{\boldsymbol{u}}+\boldsymbol{\nabla} \beta \times \overline{\boldsymbol{u}}),
$$

where the $\alpha$ is a constant with the length dimension. The time mean condition $\overline{\boldsymbol{u}}^{\prime}=0$, which requires $\bar{\beta}=0$ and $\overline{\nabla \beta}=0$.

In the Navier-Stokes equation of incompressible flow, there are four physical quantities, namely flow density $\rho$, dynamic viscosity $\mu$, flow velocity $\boldsymbol{u}$ and the flow pressure field $p$. Due to the incompressibility, the pressure field $p$ can be represented by $\nabla^{2} p=-\rho \boldsymbol{\nabla} \cdot(\boldsymbol{u} \cdot \boldsymbol{\nabla} \boldsymbol{u})$; therefore, we have a total of three independent physical quantities, namely $\rho, \mu$ and $\boldsymbol{u}$. According to Buckingham's $\Pi$ theorem [38-43], the three physical quantities, $\rho, \mu$ and $\boldsymbol{u}$, 
should produce one dimensionless $\Pi$. However, there is no dimensionless quantity $\Pi$ that can be constructed by $\rho, \mu$ and $\boldsymbol{u}$, which indicates that $\rho, \mu$ and $\boldsymbol{u}$ will participate within the turbulence in other ways. From dimensional perspectives, the three quantities, namely $\rho, \mu$ and $\overline{\boldsymbol{u}}$, can produce only one quantity, $\frac{\mu}{\rho \overline{\boldsymbol{u}}}$, having length dimensions.

Without a loss of generality, one can propose that $\alpha$ takes the form, $\alpha=\frac{\mu}{\rho U}$, where $U$ is a flow characteristic velocity. Hence, the velocity fluctuation can be expressed as:

$$
\begin{aligned}
\boldsymbol{u}^{\prime} & =\frac{\mu}{\rho U} \boldsymbol{\nabla} \times[\overline{\boldsymbol{u}} \beta(\boldsymbol{x}, t)] \\
& =\frac{\mu}{\rho U}(\beta \boldsymbol{\nabla} \times \overline{\boldsymbol{u}}+\nabla \beta \times \overline{\boldsymbol{u}}) \\
& =\frac{\mu}{\rho U}(\beta \overline{\boldsymbol{\omega}}+\nabla \beta \times \overline{\boldsymbol{u}}) .
\end{aligned}
$$

The beauty of the velocity fluctuation in Eq. (14) is that both the incompressibility $\boldsymbol{\nabla} \cdot \boldsymbol{u}^{\prime}=0$ and time average conditions $\overline{\boldsymbol{u}}^{\prime}=\lim _{T \rightarrow \infty} \frac{1}{T} \int_{t}^{t+T} \boldsymbol{u}^{\prime} d t=0$ can be satisfied simultaneously under those time mean conditions $\bar{\beta}=0$ and $\overline{\nabla \beta}=0$.

Because of no clear scientific definition of turbulence, the candidates of $\beta(\boldsymbol{x}, t)$ could be any one as long as the above conditions are satisfied. For instance, if we have experimental data on the velocity fluctuation, we can use them as the function, $\beta$.

The following shows that formulation of an approximate modelling (if exact turbulence theory does exist), does not need to know the function itself, but rather the mean value $\overline{\beta^{2}}$.

\section{THE AVERAGED REYNOLDS STRESS TENSOR}

The averaged Reynolds stress tensor $\boldsymbol{\tau}=-\rho \overline{\boldsymbol{u}^{\prime} \otimes \boldsymbol{u}^{\prime}}$ can be obtained as follows [45]:

$$
\begin{aligned}
\boldsymbol{\tau} & =-\frac{\mu^{2}}{\rho U^{2}} \overline{[\nabla \times(\beta \overline{\boldsymbol{u}})] \otimes[\nabla \times(\beta \overline{\boldsymbol{u}})]} \\
& =-\frac{\mu^{2}}{\rho U^{2}}\left(\overline{\beta^{2}} \overline{\boldsymbol{\omega}} \otimes \overline{\boldsymbol{\omega}}+\overline{(\boldsymbol{\nabla} \beta \times \overline{\boldsymbol{u}})} \otimes(\boldsymbol{\nabla} \beta \times \overline{\boldsymbol{u}})\right) \\
& =-\frac{\mu^{2}}{\rho U^{2}}\left(\overline{\beta^{2}} \overline{\boldsymbol{\omega}} \otimes \overline{\boldsymbol{\omega}}+{\overline{(\boldsymbol{\nabla} \beta \otimes \boldsymbol{\nabla} \beta)_{\times}}}_{\times}(\overline{\boldsymbol{u}} \otimes \overline{\boldsymbol{u}})\right) .
\end{aligned}
$$

Regarding the time mean quantity $\overline{\beta^{2}}$, since the velocity fluctuations move up and down around the mean velocity, the total area under the velocity fluctuation equals zero, which is similar to the cosine function, hence we can set $\overline{\beta^{2}}=1 / 2$.

Similarly, one can assume the gradient of $\beta(\boldsymbol{x}, t)$ as a sine-like function. Since $\nabla \beta \otimes \nabla \beta$ is a second homoge- nous order tensor, $\boldsymbol{\nabla} \beta \otimes \nabla \beta$ is the same in direction$\mathrm{s}$, thus, it can be expressed as a scalar (the square of the sine-like function) that multiplies the identity tensor $\boldsymbol{I}=\delta_{i j} \boldsymbol{e}_{i} \boldsymbol{e}_{j}$; therefore, considering the account of dimensions, its mean value is $\overline{(\boldsymbol{\nabla} F \otimes \boldsymbol{\nabla} \beta)}=1 / 2(\rho U / \mu)^{2} \boldsymbol{I}$. Note $\boldsymbol{I}_{\times}^{\times}(\overline{\boldsymbol{u}} \otimes \overline{\boldsymbol{u}})=\boldsymbol{I} \operatorname{tr}(\overline{\boldsymbol{u}} \otimes \overline{\boldsymbol{u}})-(\overline{\boldsymbol{u}} \otimes \overline{\boldsymbol{u}})^{T}$. [46] Finally, the averaged Reynolds stress can be proposed as follows:

$$
\boldsymbol{\tau}=\frac{1}{2} \rho(\overline{\boldsymbol{u}} \otimes \overline{\boldsymbol{u}})-\frac{1}{2} \rho \boldsymbol{I} \operatorname{tr}(\overline{\boldsymbol{u}} \otimes \overline{\boldsymbol{u}})-\frac{1}{2} \frac{\mu^{2}}{\rho U^{2}} \overline{\boldsymbol{\omega}} \otimes \overline{\boldsymbol{\omega}} .
$$

The denoted the velocity field $\boldsymbol{u}=(u, v, w)$, thus we have $\operatorname{tr}(\overline{\boldsymbol{u}} \otimes \overline{\boldsymbol{u}})=u^{2}+v^{2}+w^{2}$; therefore the last term in Eq.(16) is kinetic energy density.

This expression of the averaged Reynolds stress tensor reveals that the mean vorticity $\bar{\omega}$ is a key source in producing turbulence, and it is worth commenting here in this regard. Eq.(16) indicates clearly that the averaged Reynolds stress tensor is proportional to the square of the mean vorticity. The non-linearity between the averaged Reynolds stress tensor and the mean vorticity and velocity is the key feature of turbulence phenomena, and is totally different from molecular diffusivity [2]. Thus, non-linearity of the averaged Reynolds stress tensor is the turbulence mechanism behind rapid mixing.

\section{SIMPLIFIED CLOSED \\ REYNOLDS-CHOU-NAVIER-STOKES (RCNS) EQUATIONS}

With the explicit velocity fluctuation in Eq. (14), one can formulate the Reynolds averaged Navier-Stokes equations as follows:

$$
\begin{aligned}
\rho \nabla \cdot(\overline{\boldsymbol{u}} \otimes \overline{\boldsymbol{u}}) & =-\nabla \bar{p}+\mu \nabla^{2} \overline{\boldsymbol{u}}+\nabla \cdot \boldsymbol{\tau} \\
\nabla \cdot \overline{\boldsymbol{u}} & =0 .
\end{aligned}
$$

where the divergence of the Reynolds stress tensor is given by

$$
\begin{aligned}
\boldsymbol{\nabla} \cdot \boldsymbol{\tau} & =-\frac{1}{2} \frac{\mu^{2}}{\rho U^{2}} \overline{\boldsymbol{\omega}} \cdot \nabla \overline{\boldsymbol{\omega}} \\
& +\frac{1}{2} \rho \overline{\boldsymbol{u}} \cdot \nabla \overline{\boldsymbol{u}}-\frac{1}{2} \rho \boldsymbol{\nabla} \cdot[\boldsymbol{I} \operatorname{tr}(\overline{\boldsymbol{u}} \otimes \overline{\boldsymbol{u}})] .
\end{aligned}
$$

It is clear that Eq. (17) is a closed equation, in which the mean velocity field $\overline{\boldsymbol{u}}$ is the only unknown vector.

The above formulations show that although the specific expression of the function $F(\boldsymbol{x}, t)$ is not known, one can still approximately calculate the Reynolds stress tensor $\boldsymbol{\tau}$, the mean velocity $\overline{\boldsymbol{u}}$, mean pressure $\bar{p}$, as well as the kinetic energy of the velocity fluctuation. However, the pressure fluctuation and the vorticity fluctuation 
cannot be determined without knowing $F(\boldsymbol{x}, t)$. This imperfection does not affect the turbulence study too much, since one of the central issues is to find mean field quantities such as the mean velocity and the pressure, which can been formulated within the current theoretical framework.

\section{FORMULATIONS IN CARTESIAN COORDINATES}

For a better understanding, the formulations in Cartesian coordinates are listed below:

The vorticity components are: $\bar{\omega}_{x}=\frac{\partial \bar{w}}{\partial y}-\frac{\partial \bar{v}}{\partial z}, \bar{\omega}_{y}=$ $\frac{\partial \bar{u}}{\partial z}-\frac{\partial \bar{w}}{\partial x}$, and $\bar{\omega}_{z}=\frac{\partial \bar{v}}{\partial x}-\frac{\partial \bar{u}}{\partial y}$.

The velocity fluctuation components are:

$$
\begin{aligned}
u^{\prime} & =\frac{\mu}{\rho U} \beta(\boldsymbol{x}, t)\left(\frac{\partial \bar{w}}{\partial y}-\frac{\partial \bar{v}}{\partial z}\right), \\
v^{\prime} & =\frac{\mu}{\rho U} \beta(\boldsymbol{x}, t)\left(\frac{\partial \bar{u}}{\partial z}-\frac{\partial \bar{w}}{\partial x}\right), \\
w^{\prime} & =\frac{\mu}{\rho U} \beta(\boldsymbol{x}, t)\left(\frac{\partial \bar{v}}{\partial x}-\frac{\partial \bar{u}}{\partial y}\right) .
\end{aligned}
$$

The averaged Reynolds stress components are:

$$
\begin{aligned}
\tau_{x x} & =-\frac{1}{2} \rho\left(\bar{v}^{2}+\bar{w}^{2}\right)-\frac{1}{2} \frac{\mu^{2}}{\rho U^{2}}\left(\frac{\partial \bar{w}}{\partial y}-\frac{\partial \bar{v}}{\partial z}\right)^{2} \\
\tau_{x y} & =\frac{1}{2} \rho \bar{u} \bar{v}-\frac{1}{2} \frac{\mu^{2}}{\rho U^{2}}\left(\frac{\partial \bar{w}}{\partial y}-\frac{\partial \bar{v}}{\partial z}\right)\left(\frac{\partial \bar{u}}{\partial z}-\frac{\partial \bar{w}}{\partial x}\right), \\
\tau_{x z} & =\frac{1}{2} \rho \bar{u} \bar{w}-\frac{1}{2} \frac{\mu^{2}}{\rho U^{2}}\left(\frac{\partial \bar{w}}{\partial y}-\frac{\partial \bar{v}}{\partial z}\right)\left(\frac{\partial \bar{v}}{\partial x}-\frac{\partial \bar{u}}{\partial y}\right), \\
\tau_{y x} & =\tau_{x y}, \\
\tau_{y y} & =-\frac{1}{2} \rho\left(\bar{u}^{2}+\bar{w}^{2}\right)-\frac{1}{2} \frac{\mu^{2}}{\rho U^{2}}\left(\frac{\partial \bar{u}}{\partial z}-\frac{\partial \bar{w}}{\partial x}\right)^{2}, \\
\tau_{y z} & =\frac{1}{2} \rho \bar{v} \bar{w}-\frac{1}{2} \frac{\mu^{2}}{\rho U^{2}}\left(\frac{\partial \bar{u}}{\partial z}-\frac{\partial \bar{w}}{\partial x}\right)\left(\frac{\partial \bar{v}}{\partial x}-\frac{\partial \bar{u}}{\partial y}\right), \\
\tau_{z x} & =\tau_{x z}, \\
\tau_{z y} & =\tau_{y z}, \\
\tau_{z z} & =-\frac{1}{2} \rho\left(\bar{u}^{2}+\bar{v}^{2}\right)-\frac{1}{2} \frac{\mu^{2}}{\rho U^{2}}\left(\frac{\partial \bar{v}}{\partial x}-\frac{\partial \bar{u}}{\partial y}\right)^{2} .
\end{aligned}
$$

It is clear that the Reynolds stress is proportional to the square mean velocity gradient, which has been proved by experiments. Tennekes and Lumley [2] pointed out that diagonal components of $\tau$, their values $\rho u_{1}^{2}, \rho u_{2}^{2}, \rho u_{3}^{2}$, in many flows, contribute little to the transport of mean momentum. The off-diagonal components of $\tau$ are shear stresses; they play a dominate role in the theory of mean momentum transfer by turbulent motion. Therefore the diagonal components of the Reynolds stresses, $\rho u_{1}^{2}, \rho u_{2}^{2}, \rho u_{3}^{2}$, can be omitted.
The Reynolds Navier-Stokes equations are:

$$
\begin{aligned}
& \bar{u} \frac{\partial \bar{u}}{\partial x}+\bar{v} \frac{\partial \bar{u}}{\partial y}+\bar{w} \frac{\partial \bar{u}}{\partial z}+\frac{1}{\rho} \frac{\partial \bar{p}}{\partial x}=\nu \nabla^{2} \bar{u}-(\boldsymbol{\nabla} \cdot \boldsymbol{\tau})_{x}, \\
& \bar{u} \frac{\partial \bar{v}}{\partial x}+\bar{v} \frac{\partial \bar{v}}{\partial y}+\bar{w} \frac{\partial \bar{v}}{\partial z}+\frac{1}{\rho} \frac{\partial \bar{p}}{\partial x}=\nu \nabla^{2} \bar{u}-(\boldsymbol{\nabla} \cdot \boldsymbol{\tau})_{y}(22) \\
& \bar{u} \frac{\partial \bar{w}}{\partial x}+\bar{v} \frac{\partial \bar{w}}{\partial y}+\bar{w} \frac{\partial \bar{w}}{\partial z}+\frac{1}{\rho} \frac{\partial \bar{p}}{\partial x}=\nu \nabla^{2} \bar{u}-(\boldsymbol{\nabla} \cdot \boldsymbol{\tau})_{z},
\end{aligned}
$$

where the kinematic viscosity is $\nu=\mu / \rho$, and the Laplace operator $\nabla^{2}=\frac{\partial^{2}}{\partial x^{2}}+\frac{\partial^{2}}{\partial y^{2}}+\frac{\partial^{2}}{\partial z^{2}}$, and $(\boldsymbol{\nabla} \cdot \boldsymbol{\tau})_{x}=\frac{\partial \tau_{x x}}{\partial x}+$ $\frac{\partial \tau_{x y}}{\partial y}+\frac{\partial \tau_{x z}}{\partial z},(\boldsymbol{\nabla} \cdot \boldsymbol{\tau})_{y}=\frac{\partial \tau_{y x}}{\partial x}+\frac{\partial \tau_{y y}}{\partial y}+\frac{\partial \tau_{y z}}{\partial z},(\boldsymbol{\nabla} \cdot \boldsymbol{\tau})_{z}=$ $\frac{\partial \tau_{z x}}{\partial x}+\frac{\partial \tau_{z y}}{\partial y}+\frac{\partial \tau_{z z}}{\partial z}$.

\section{DISCUSSIONS}

(1) The flow characteristic velocity $U$ has proved that it can be chosen for any given problem. For flow speed that travels close to the speed of sound $c$, one can choose the speed of sound $c$ as the flow characteristic velocity $U$, namely $U=c$. The sound waves can exist in a fluid without the presence of any external force field, which propagates by restoring force that is provided entirely by the fluid's own compressibility [44]; therefore, the velocity fluctuation conjecture in Eq.(14) will be intrinsic if the speed of the sound waves is chosen as the flow characteristic velocity $U$.

(2) Since the velocity fluctuation is proportional to the vorticity $\overline{\boldsymbol{\omega}}=\boldsymbol{\nabla} \times \overline{\boldsymbol{u}}$, and Curl of the mean velocity, $\boldsymbol{\nabla} \times \overline{\boldsymbol{u}}$ is a three dimensional quantity; therefore, the turbulence is always rotational and three dimensional, whilst characterized by high levels of fluctuation vorticity. Hence, vorticity dynamics play an essential role in the description of turbulent velocity fluctuations [2].

(3) If the scalar function $\beta$ is only a function of time, the above formulations can be further simplified as follows:

$$
\boldsymbol{u}^{\prime}=\frac{\mu}{\rho U} \beta \overline{\boldsymbol{\omega}}
$$

The Reynolds stress tensor is given by

$$
\boldsymbol{\tau}=-\frac{1}{2} \frac{\mu^{2}}{\rho U^{2}} \overline{\boldsymbol{\omega}} \otimes \overline{\boldsymbol{\omega}} .
$$

In this special case, the Reynolds averaged stress tensor is produced fully by the mean vorticity.

(4) Similarly, compressible flow temperature fluctuation $T^{\prime}$ and density fluctuation $\rho^{\prime}$ should also be constructed. 


\section{CONCLUSIONS}

In summary, this study has attempted to propose a simplification of the velocity fluctuations that can simultaneously satisfy both incompressibility and time-average conditions. The simplified closed Reynolds Navier-Stokes turbulence formulations show that the mean vorticity has a strong influence on the velocity fluctuation and the Reynolds stress tensor, as well as on the mean pressure. This fact reveals that three-dimensional vorticity fluctuation is a fundamental mechanism to producing turbulence.

\section{Acknowledgements}

It is my great pleasure to have shared and discussed some of the above with Michael Sun from Bishops Diocesan College, whose pure and direct scientific sense inspired me.

* Electronic address: sunb@cput.ac.za

[1] Bradshaw P. An Introduction to Turbulence and Its Measurement. Pergamon Press, New York (1971)

[2] Tennekes, H. and Lumley, J.L. A First Course in Turbulence. Cambridge: The MIT Press (1972).

[3] Lesilie D.C. Developments in the Theory of Turbulence. Clarendon Press, Oxford (1973).

[4] Townsend A.A. The Structure of Turbulent Shear Flow 2nd ed., Cambridge University Press, New York (1976).

[5] Lesieur M. Turbulence in Fluids. 2nd ed. Kluwer, Dordrecht (1990).

[6] Wilcox D.C. Turbulence Modeling for CFD. D C W Industries (1993).

[7] Pope S.B. Turbulent Flows. Cambridge University Press, Cambridge (2000).

[8] Davidson, P.A. Turbulence. Oxford University Press, Oxford (2004).

[9] Hof B. Experimental Observation of Nonlinear Traveling Waves in Turbulent Pipe Flow. Science 305, 1594 (2004)

[10] Falkovich, G. and Sreenivasan K.R. Lessons from hydrodynamic turbulence. Physics Today, 43-49 (April 2006).

[11] Frisch, U. Turbulence: The Legacy of A.N. Kolmogorov. Cambridge University Press, Cambridge (2008).

[12] Marusic I. Mathis R. and Hutchins N. Predictive model for wall-bounded turbulent Flow. Science 329, 193 (2010).

[13] Smits A.J., McKeon B.J. and Marusic I. High - Reynolds Number Wall Turbulence, Annu. Rev. Fluid Mech. 43, 353 (2011).

[14] Davison, P.A. et al. A Voyage Through Turbulence. Cambridge: Cambridge University Press (2011)
[15] Suri B., Tithof J. R., Grigoriev R.O. and Schatz M.F. Forecasting Fluid Flows Using the Geometry of Turbulence. Phys. Rev. Lett. 118, 114501 (2017).

[16] Cardesa et al. The turbulent cascade in five dimensions. Science 357, $782-784$ (2017) 25

[17] http://www.claymath.org/millennium-problems/navierstokes-equation.

[18] Castelvecchi, D. On the trial of turbulence. Nature, 548:382 (2017).

[19] Kolmogorov, A.N. The local structure of turbulence in incompressible viscous fluid for very large Reynolds number. Dokl. Akad. Nauk SSSR, 30:299-303 (1941a) (reprinted in Proc.R.Soc.Lond. A, 434,9-13, 1991).

[20] Kolmogorov, A.N. On degeneration (decay) of isotropic turbulence in an incompressible visous liquid. Dokl. Akad. Nauk SSSR, 31:538-540 (1941b).

[21] Kolmogorov, A.N. Dissipation of energy in locally isotropic turbulence. Dokl.Akad. Nauk SSSR, 32:1618 (1941c).(reprinted in Proc.R.Soc.Lond. A, 434,15-17, 1991).

[22] Sreenivasan K.R. On the scaling of the turbulence energy dissipation rate. Physics of Fluids, 27,5:1048-1051 (1984).

[23] Sun, B. The temporal scaling laws of compressible turbulence. Modern Physics Letters B. 30,(23) 1650297 (2016).

[24] Sun, B. Scaling laws of compressible turbulence. Appl. Math. Mech.-Engl. Ed. 38: 765(2017).

[25] She Z. S. and Lévêque E. Universal scaling laws in fully developed turbulence. Phys. Rev. Lett. 72,336(1994) .

[26] Lorenz E.N. Deterministic non-periodic flow. J. Atmos Sci. 20:130 - 41(1963).

[27] Benzi R, Paladin P., Parisis G. and Vulpiani A. On the multifractal nature of fully developed turbulence and chaotic systems. J. Phys. A: Math. Gen. 17:35213531(1984).

[28] Orszag S. A. and Patterson G. S. Jr. Numerical simulation of three-dimensional homogeneous isotropic turbulence. Phys. Rev. Lett. 28,76(1972).

[29] Reynolds O. On the dynamical theory of incompressible viscous fluids and the determination of the criterion. Philos. Trans. R. Soc. 186:123 - 164(1895).

[30] Chou P-Y. On an extension of Reynolds' method of finding apparent stress and the nature of turbulence. Chin. J. Phys. 1-53 (1940).

[31] Chou P-Y. On velocity correlations and the solutions of the equations of turbulent fluctuation. Q. Appl. Math. 111(1):38-54(1945).

[32] Chou, P-Y, Cai S-T. The vorticity structure of homogeneous isotropic turbulence in its final period of decay. Acta Mech. Sinica 1(1): 3-14(1957).

[33] Chou, P-Y, Huang Y-N. On the statistical vorticity structure theory of homogeneous isotropic turbulence. Sci. Sinica XVIII(2): 199-222(1975).

[34] Chou P-Y. On the condition of pseudo-similarity and the theory of turbulence. Sci. Sinica XXVIII(4): 405(1985)

[35] Chou, P-Y, Chen S-Y. On the theory of turbulence for incompressible fluid. Sci. Sinica (4): 369-80 (1987).

[36] Chou, P-Y. and Ru-Lin Chou, 50 years of turbulence re- 
search in China. Annu. Rev. Fluid Mech. 27:1-15(1995).

[37] Sun, B. The Reynolds Navier-Stokes Turbulence Equations of Incompressible Flow Are Closed Rather Than Unclosed. Preprints 2018, 2018060461 (doi: 10.20944/preprints201806.0461.v1).

[38] Buckingham E. Notes on the method of dimensions. Phil. Mag. 42:696 - 719 (1921).

[39] Bridgman P.W. Dimensional Analysis. Yale University Press, New Haven (1922)

[40] Sedov L.I. Similarity and Dimensional Analysis in Mechanics. Academic Press, New Yrok (1959)

[41] Barenblatt G.I. Similarity, Self-similarity and Intermediate Asymptotics. Cambridge University Press, Cam- bridge (1996)

[42] Sun B. Dimensional Analysis and Lie Group. (China High Education Press, Beijing, 2016).

[43] Hecksher, T. Insights through dimensions. Nature Physics, 13, 2017.

[44] Lighthill, J. Wavews in Fluids. Cambdrige University Press, Cambdridge (1978).

[45] For tensors $\boldsymbol{A}, \boldsymbol{B}, \boldsymbol{C}, \boldsymbol{D}$, where there is tensor identity $(\boldsymbol{A} \times \boldsymbol{B}) \otimes(\boldsymbol{C} \times \boldsymbol{D})=(\boldsymbol{A} \otimes \boldsymbol{C})_{\times}^{\times}(\boldsymbol{B} \otimes \boldsymbol{D})$ and $(\boldsymbol{\nabla} \times \boldsymbol{B}) \otimes$ $(\nabla \times D)=(\nabla \boldsymbol{A})_{\times}^{\times}(\nabla \boldsymbol{B})$.

[46] For a tensor $\boldsymbol{A}$ and identity tensor $\boldsymbol{I}=\delta_{i j} \boldsymbol{e}_{i} \boldsymbol{e}_{j}$, and the identity $\boldsymbol{I}_{\times}^{\times} \boldsymbol{A}=\boldsymbol{I} \operatorname{tr}(\boldsymbol{A})-\boldsymbol{A}^{T}$. 